\title{
Solar coronal rotation determined by $X$-ray bright points in Hinode/XRT and Yohkoh/SXT full-disc images
}

\author{
R. Kariyappa
}

Indian Institute of Astrophysics, Bangalore 560 034, India

e-mail: rkari@iiap.res.in

Received 18 February 2008 / Accepted 6 May 2008

\section{ABSTRACT}

\begin{abstract}
Aims. Our aim is to identify and trace the X-ray bright points (XBPs) over the disc and use them as tracers to determine the coronal rotation. This investigation will help to clarify and understand several issues: (i) whether the corona rotates differentially; (ii) whether the rotation depends on the sizes of the XBPs; and (iii) whether there is a dependence on phases of the solar magnetic cycle.

Methods. We analysed the daily full-disc soft X-ray images observed with (i) X-Ray Telescope (XRT) onboard the Hinode mission during January, March, and April 2007; and (ii) Soft X-ray Telescope (SXT) onboard the Yohkoh from 1992 to 2001 using SSW in IDL. We used the tracer method to trace the passage of XBPs over the solar disc with the help of overlaying grids and derived the sidereal angular rotation velocity and the coordinates (latitude and longitude) of the XBPs.

Results. We have determined the position of a large number of XBPs, both in Hinode/XRT and Yohkoh/SXT images, and followed them over the solar disc as a function of time. We derived the coronal sidereal angular rotation velocity and compared it with heliocentric latitude and as a function of solar activity cycle. In addition, we measured the sizes of all the XBPs and related them to the coronal rotation. The important results derived from these investigations are: (i) the solar corona rotates differentially like the photosphere and chromosphere; (ii) the sidereal angular rotation velocity is independent of the sizes of the XBPs; (iii) the sidereal angular rotation velocity does not depend on phases of the solar magnetic cycle; and (iv) the differential rotation of the corona is present throughout the solar magnetic cycle.
\end{abstract}

Key words. Sun: corona - Sun: rotation - Sun: magnetic fields - Sun: X-rays, gamma rays

\section{Introduction}

The Sun rotates differentially (i.e. the equatorial regions rotate faster than the polar regions) at the photosphere and chromosphere. The measurements of solar rotation have been carried out by two methods, namely, (i) tracer method by tracing the passage of various features like sunspots, faculae, filaments, etc., over the solar disc, and (ii) Doppler method - by the spectroscopic observations of Doppler displacements of the core of the spectral lines. The phenomenon of solar rotation is still to be understood clearly from the existing large volumes of data. On the other hand, the coronal rotation is observed through features like Fe XIV green lines, soft X-rays, and radio waves. The coronal rotation has been measured by two methods: (i) tracer method based on visual tracing of coronal features (XBPs) in consecutive images and (ii) automatic method - relies on the IDL procedure "regions of interest" segmentation that is used to identify and follow them in the consecutive images. However, in both the methods, there are advantages and disadvantages. The coronal rotation determination appears to be more complicated and even less understood, because the corona is optically thin across a wide range of observed wavelengths, and the features are less distinct in duration and extent.

Solar coronal X-ray bright points (XBPs) were discovered using a soft X-ray telescope (SXT) on a sounding rocket, and their nature has been an enigma since their discovery in the late 1960's (Vaiana et al. 1970). Later, using Skylab and Yohkoh X-ray images, the XBPs were studied in great detail (Golub et al. 1974; Harvey 1996; Nakakubo \& Hara 1999; Longcope et al. 2001; Hara \& Nakakubo 2003). It has been shown that the XBPs have a spatial correspondence with small-scale bipolar magnetic regions by comparing the ground-based magnetic field measurements with simultaneous space-borne X-ray imaging observations (Krieger et al. 1971; Golub et al. 1977). The number of XBPs (daily) found on the Sun varies from several hundreds up to a few thousand (Golub et al. 1974). Zhang et al. (2001) find a density of 800 XBPs for the entire solar surface at any given time. It is known that the observed XBP number is anticorrelated with the solar cycle, but this is an observational bias, and the number density of XBPs is nearly independent of the $11 \mathrm{yr}$ solar activity cycle (Nakakubo \& Hara 1999; Sattarov et al. 2002; Hara \& Nakakubo 2003). Golub et al. (1974) found that the diameters of the XBPs are around 10-20 arcsec, and their lifetimes range from $2 \mathrm{~h}$ to 2 days (Zhang et al. 2001). The physical studies have indicated the temperatures are fairly low, $T=2 \times 10^{6} \mathrm{~K}$, and the electron densities $n_{\mathrm{e}}=5 \times 10^{9} \mathrm{~cm}^{-3}$ (Golub \& Pasachoff 1997). Recently, Kariyappa \& Varghese (2008) have analysed the time sequence of XBPs in soft X-ray images obtained from Hinode/XRT and discuss the nature of intensity oscillations associated with the different classes of XBPs showing the different emission levels. It has been shown by various groups that the solar corona rotates differentially on the basis of their analysis of coronal bright points in SOHO/EIT images (Brajša et al. 2001, 2002, 2004; Karachik et al. 2006) and by using radio observations at different frequencies (Vats et al. 2001). However, further investigations are required to confirm the differential rotation of the corona. In particular it is not clear that the sidereal angular rotation velocity of the corona depends on the sizes and lifetimes of the tracers (XBPs) and how it varies with the phases of the solar cycle. 
Table 1. Variation in the coefficients $A, B$ and their standard errors $E A$ and $E B$ in deg/day for different tracers.

\begin{tabular}{ccccc}
\hline \hline Tracer & $A \pm E A$ & $-B \pm E B$ & Data source & References \\
\hline XBPs & $14.192 \pm 0.170$ & $4.211 \pm 0.775$ & Hinode/XRT & present work \\
XBPs & $17.597 \pm 0.398$ & $4.542 \pm 1.136$ & Yohkoh/SXT & present work \\
Coronal bright points & $14.677 \pm 0.033$ & $3.100 \pm 0.140$ & SOHO/EIT & Brajša et al. (2004) \\
Sunspots & $14.551 \pm 0.006$ & $2.870 \pm 0.060$ & Greenwich & Balthasar et al. (1986) \\
Sunspots & $14.370 \pm 0.010$ & $2.590 \pm 0.160$ & Greenwich & Brajša et al. (2002) \\
\hline
\end{tabular}

In this paper, we analysed two sets of observations of full-disc soft X-ray images obtained from Hinode/XRT and Yohkoh/SXT experiments to determine the coronal rotation using the XBPs as tracers and explore the evidence for differential rotation and its relation to the sizes of the XBPs and phases of the solar magnetic cycle.

\section{Observations and methods of data analysis}

\subsection{Hinode/XRT full-disc images}

We used daily full-disc soft X-ray images from the period of January, March, and April 2007, from the X-Ray Telescope (XRT) onboard the Hinode mission. The images were observed through a single X-ray Ti_poly filter, and the image size is $2048^{\prime \prime} \times 2048^{\prime \prime}$ with a spatial resolution of $1.032^{\prime \prime} /$ pixel. All the full-disc X-ray images were visually inspected and the XBPs identified in image sequences. The main criterion for identifying a XBP was to check its persistence in the consecutive images at approximately the same latitude and shifted in the central meridian distance (CMD) according to the elapsed time. The CMD values of the identified features were then measured in selected consecutive images and were fitted as a function of time. The correlation coefficient of the function CMD was generally very high, implying that the tracers were correctly identified. We identified and selected a isolated and distinct 63 XBPs that are distributed over the different heliographic latitudes. Using SSW in IDL, we generated the full-disc maps of the images and overlayed them with longitude and latitude grid maps. Using the tracer method (based on a visual identification of a particular $\mathrm{XBP}$ that can be used as a tracer), coronal X-ray bright points are visually traced in consecutive images on a computer screen. This was carried out several times. The tracing of XBPs was performed in 211 consecutive images for the observing period of January, March, and April 2007. The rotation velocities were determined by a linear least-squares fit of the central meridian distances (CMD) as a function of time, and more images were used to determine the velocity of each XBP. We measured a large number of rotation velocities for different possible CMDs in each latitude band, so that more data points were used in the analysis. We compared the angular rotational velocity values as a function of latitude of the corresponding XBPs.

\subsection{Yohkoh/SXT full-disc images}

Multiple observing sequences of the Yohkoh/SXT full-frame images selected for 3 months in each year for the time interval from 1992 to 2001 were used for the analysis. From the daily images we identified the distinct and isolated XBPs and selected 134 XBPs. The tracer method was applied to Yohkoh/SXT images (we have already discussed the details of the method above for the Hinode/XRT images) for all the XBPs as they pass over the solar disc and measured their coordinates. From these coordinates, the sidereal angular rotation velocities and their sizes in terms of arcsec for all the years of data were calculated. Finally, we made the plots of angular rotation velocity as a function of (i) latitude for all the years and separately for each year and (ii) sizes of the XBPs. Only those XBPs that were present in at least five to six consecutive images were selected for the rotation velocity and size determination from Hinode/XRT and Yohkoh/SXT data sets. Further details on the method of data analysis are also discussed below, together with the results and discussions.

\section{Results and discussions}

\subsection{Coronal differential rotation profiles}

For comparison with previous studies of solar rotation, we fit the data by $A+B \sin ^{2} \psi+C \sin ^{4} \psi$, where $\psi$ is the solar latitude and the coefficients $A, B$, and $C$ listed for Hinode/XRT and Yohkoh/SXT data in Table 1. In the additional constraint $B=C$ is assumed to avoid crosstalk between the parameters $B$ and $C$. This is especially important when the data from middle and higher solar latitudes are considered and when the parameters from different data sets are compared. However, one way to avoid crosstalk is to obtain the data from all latitudes and, in this context, XBPs covering latitudes from the equator up to about $70 \mathrm{deg}$, which has an obvious advantage. The coefficients (from Table 1) of the above approximation for the Hinode/XRT data are in general agreement with previous studies (e.g., Brajša et al. 2001a, 2002, 2004) with an equatorial rotation velocity. The equatorial rotation velocity of Yohkoh/SXT data is higher than that of Hinode/XRT rotation velocities. It is also higher than other published results of equatorial rotation from Yohkoh data (see for example the Fig. 2 of Brajša et al. 2001b). Figure 1 shows a profile of the solar rotation of 63 XBPs derived from the images of Hinode/XRT for all 3-months (January, March, and April 2007). Although there is significant scatter in the profile, the solar differential rotation is clearly seen. The scatter in Fig. 1 can be attributed to evolution and proper motions of some of the XBPs, as well as error in displacements determination. We find less data points at a higher latitude in Fig. 1, and it is mainly due to the absence of XBPs at higher latitudinal regions. Similarly, in Fig. 2a, we show the angular rotation velocity for all the 134 XBPs as a function of latitude derived from Yohkoh/SXT images for the years from 1992 to 2001. We find that the latitudinal profile of solar rotation derived from XBPs in Hinode/XRT images is similar to the one derived independently from XBPs of Yohkoh/SXT full-disc images, and the differential rotation is clearly seen in both cases. In addition, we infer from Figs. 1 and $2 \mathrm{a}$ that the equatorial sidereal angular rotation velocity in the case of Yohkoh/SXT (17.6 deg/day from Table 1) is higher than in Hinode XBPs (14.2 deg/day), coronal bright points (14.7 deg/day, Brajša et al. 2004), and sunspot regions (14.5 deg/day) at the photospheric level (Howard 1984; Balthasar et al. 1986). However, the reason for the presence of higher rotational velocity with Yohkoh/SXT data compared to other results is not understood. 


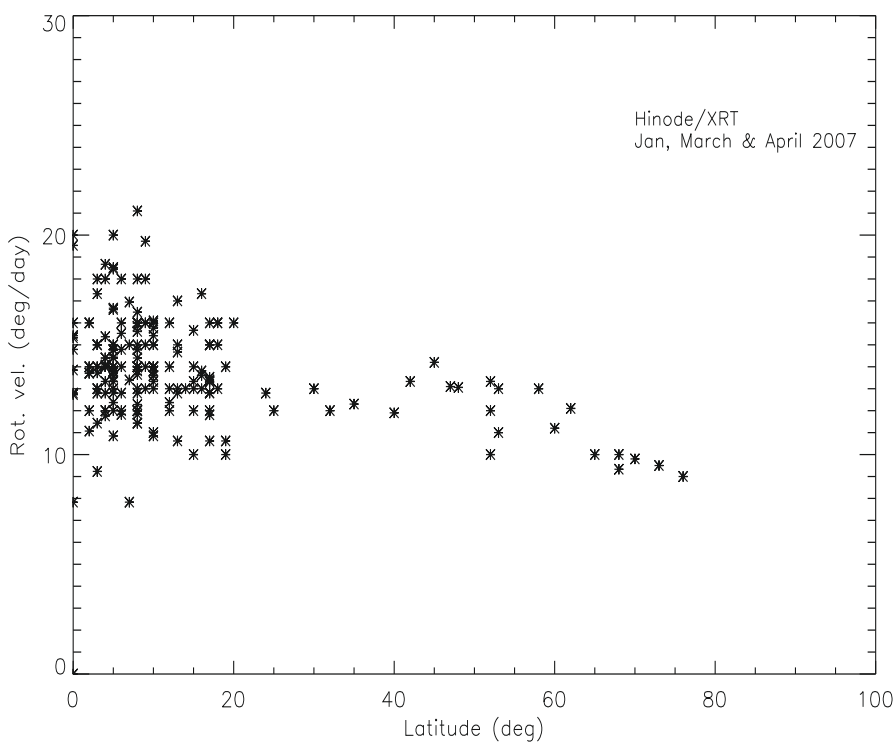

Fig. 1. Coronal sidereal angular rotation velocity as a function of latitude to demonstrate that the corona rotates differentially. The rotation velocity has been measured with the help of XBPs as tracers from Hinode/XRT full-disc images of January, March, and April 2007.

\subsection{Dependence of coronal differential rotation on the sizes of XBPs}

In addition to the sidereal angular rotation velocity of XBPs, we measured the sizes of all the 134 XBPs in full-disc images observed from Yohkoh/SXT for the time interval from 1992 to 2001. To determine the relationship between the sidereal angular rotation velocity and the sizes of the XBPs, we have plotted the sidereal angular rotation velocity as a function of latitude in Fig. 2a and as a function of sizes of the XBPs in Fig. 2b. The correlation coefficient between the sidereal angular rotation velocity and the sizes of XBPs is found to be 0.065 . It is evident from Fig. 2 that the coronal sidereal angular rotation velocity does not depend on the sizes of XBPs. At the same time, it is interesting to re-investigate how the sidereal angular rotation velocity varies in longlived and shortlived XBPs in comparison with photospheric sunspots and surroundings. We examined the life span and variations in sidereal angular rotation velocity of some of the XBPs from the collection and find that the sidereal angular rotation velocity values appear to not change with their lifetime. This means that the long- and shortlived XBPs show almost the same rotational velocity. In contrast, Golub \& Vaiana (1978) have shown from their preliminary analysis that the rotation rate of longlived X-ray features is equal to that of sunspots and, on the other hand, the shortlived X-ray features rotation rate is consistent with that of photospheric gas. In the case of sunspot data analysis, Balthasar et al. (1986) concluded that there is a tendency that larger sunspot groups will yield a higher equatorial velocities, but this tendency is much weaker than the dependence on the type of sunspots. However, the coronal sidereal angular rotation velocity does not depend on the sizes of $\mathrm{XBPs}$, and we cannot extrapolate the relationship between the photospheric rotational velocity and the sizes of sunspot groups to coronal level.

\subsection{Variation in coronal differential rotation as a function of the phases of the solar magnetic cycle}

The coronal X-ray bright points are always present throughout the solar cycle and are more broadly distributed over all the
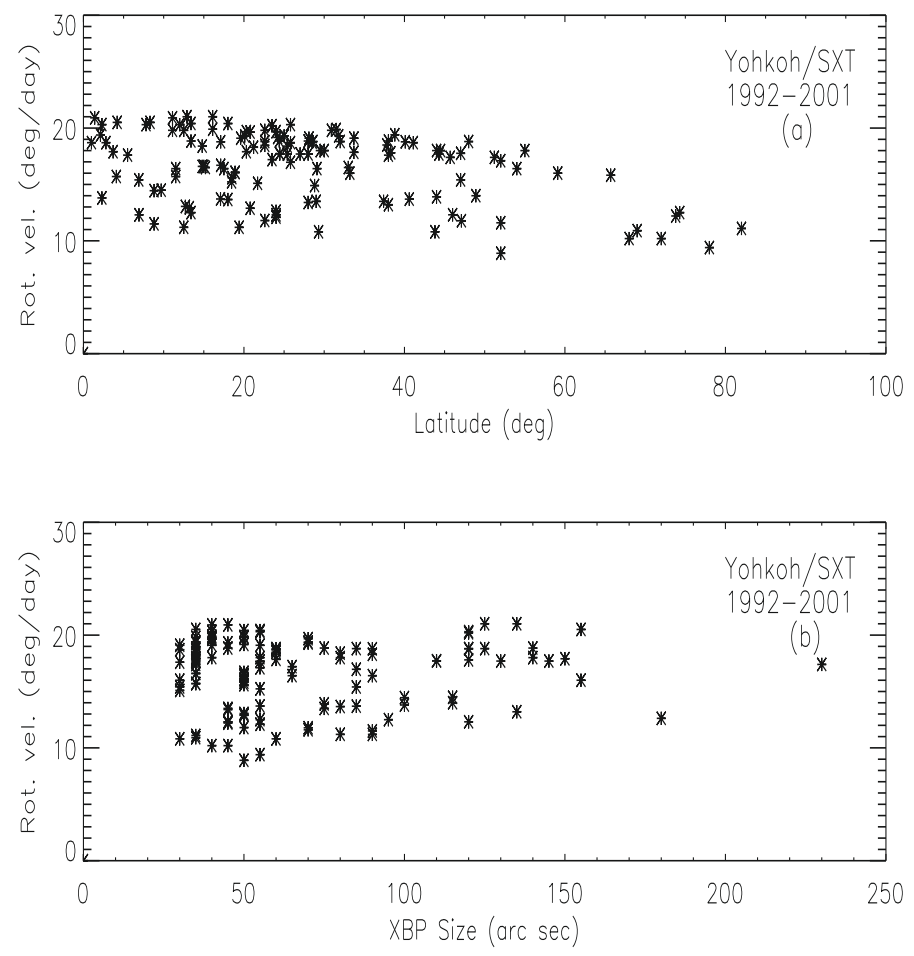

Fig. 2. Coronal sidereal angular rotation velocity is compared with (a) latitude and (b) sizes of the XBPs derived from the images of Yohkoh/SXT for the time interval from 1992 to 2001. The correlation coefficient between the rotation velocity and sizes of XBPs is 0.065 .

latitude regions compared to sunspot regions, so that the differential rotation can be measured at any phase in the solar cycle. It is known that the observed XBP number is anti-correlated with the solar cycle, but this is an observational bias and the number density of XBPs is nearly independent of the $11 \mathrm{yr}$ solar activity cycle (Nakakubo \& Hara 1999; Sattarov et al. 2002; Hara \& Nakakubo 2003). In Fig. 3, we have plotted, for Yohkoh/SXT data, the sidereal angular rotation velocity as a function of latitude for the years from 1992 and 2001 separately. Each rectangular box in Fig. 3 represents the differential rotation profile of each year. It is known that, for sunspots at the photospheric level, the mean rotation period varied from cycle to cycle in such a way that the rotation period was shorter in the low activity cycle, and there was longer rotation period in high activity cycle (Kambry \& Nishikawa 1990). On the other hand, Mehta (2005) analysed radio observational data and found that there is no systematic relationship between coronal rotation period and phase of the solar cycle. We calculated the parameters $(A$ and $B)$ of the solar coronal differential rotation for each year separately and tabulated them in Table 2. For comparison, the yearly mean sunspot numbers are also presented in Table 2. In Fig. 4, we have plotted the angular rotation velocity against the yearly mean sunspot number, and the correlation coefficient $(r)$ is found to be 0.2 . It is clearly seen from Fig. 3, Table 2, and Fig. 4 that the variation in the rotational velocities is independent of the phases of the solar magnetic cycle, and the corona rotates differentially in all the years. In the case of photospheric differential rotation using sunspots as tracers, the rotation velocity is anticorrelated with the solar activity (as shown in Fig. 2 and Table 7 of Balthasar et al. 1986).

From a detailed analysis of daily full-disc X-ray images obtained using the Hinode/XRT and Yohkoh/SXT experiments, we conclude that the coronal X-ray bright points are good tracers 

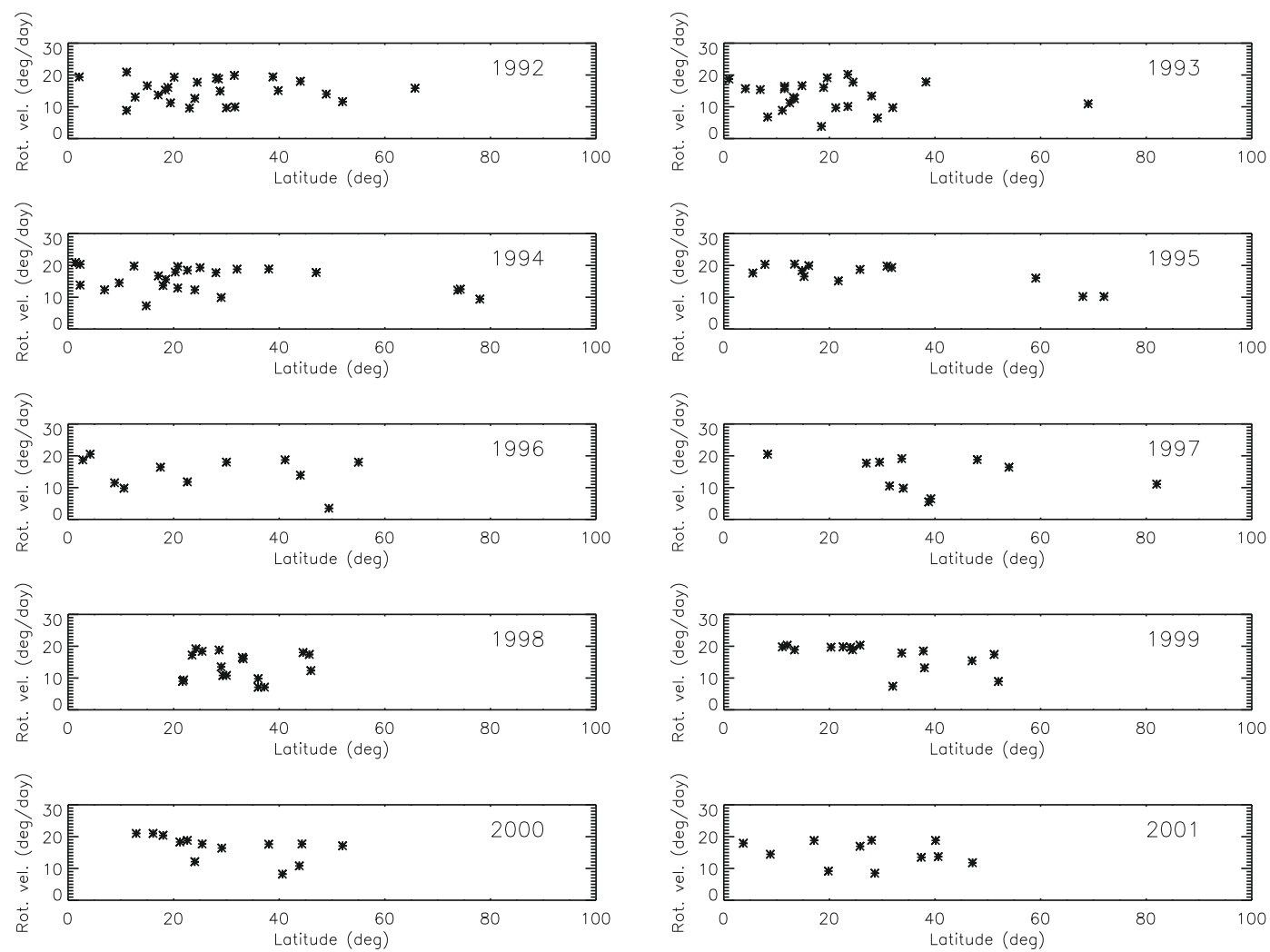

Fig. 3. Variation in coronal rotation velocity as a function of the phases of the solar magnetic cycle. The sidereal angular rotation velocities have been derived from the XBPs in Yohkoh/SXT images for time interval from 1992 to 2001.

Table 2. Variation in the coefficients $A$ and $B$ and their errors $E A$ and $E B$ in $\operatorname{deg} /$ day for different years representing different phases of the solar cycle.

\begin{tabular}{cccc}
\hline \hline Year & $A \pm E A$ & $-B \pm E B$ & $\begin{array}{c}\text { Yearly mean } \\
\text { sunspot numbers }\end{array}$ \\
\hline 1992 & $15.296 \pm 1.161$ & $0.344 \pm 3.698$ & 93.6 \\
1993 & $13.683 \pm 1.208$ & $2.698 \pm 5.203$ & 54.5 \\
1994 & $16.542 \pm 0.985$ & $4.261 \pm 2.618$ & 31.0 \\
1995 & $19.547 \pm 0.791$ & $8.663 \pm 1.873$ & 18.2 \\
1996 & $15.536 \pm 2.288$ & $3.717 \pm 6.665$ & 8.4 \\
1997 & $16.216 \pm 3.139$ & $5.638 \pm 6.754$ & 20.3 \\
1998 & $13.552 \pm 2.773$ & $-0.175 \pm 8.836$ & 62.6 \\
1999 & $20.359 \pm 1.533$ & $12.266 \pm 4.645$ & 96.1 \\
2000 & $19.618 \pm 1.768$ & $10.911 \pm 5.486$ & 123.3 \\
2001 & $15.704 \pm 2.062$ & $3.984 \pm 7.078$ & 123.3 \\
\hline
\end{tabular}

to determine the angular rotation velocity of the corona. It is found that the corona rotates differentially as in the photosphere and chromosphere. The coronal sidereal angular rotation velocity does not depend on the sizes of XBPs, and the differential rotation is present throughout the solar cycle. The results do not reveal any strong evidence of any dependence of angular rotation velocity on the solar magnetic cycle.

Acknowledgements. Hinode is a Japanese mission developed and launched by ISAS/JAXA, collaborating with NAOJ as a domestic partner with NASA and STFC (UK) as international partners. Scientific operation of the Hinode mission is conducted by the Hinode science team organised at ISAS/JAXA. This team mainly consists of scientists from institutes in the partner countries. Support for the postlaunch operation is provided by JAXA and NAOJ (Japan), STFC (UK), NASA (USA), ESA, and NSC (Norway). The SXT program was supported at Lockheed under NASA contract NAS8-37334 with the Marshall Space Flight Center and by the Lockheed Independent Research Program.

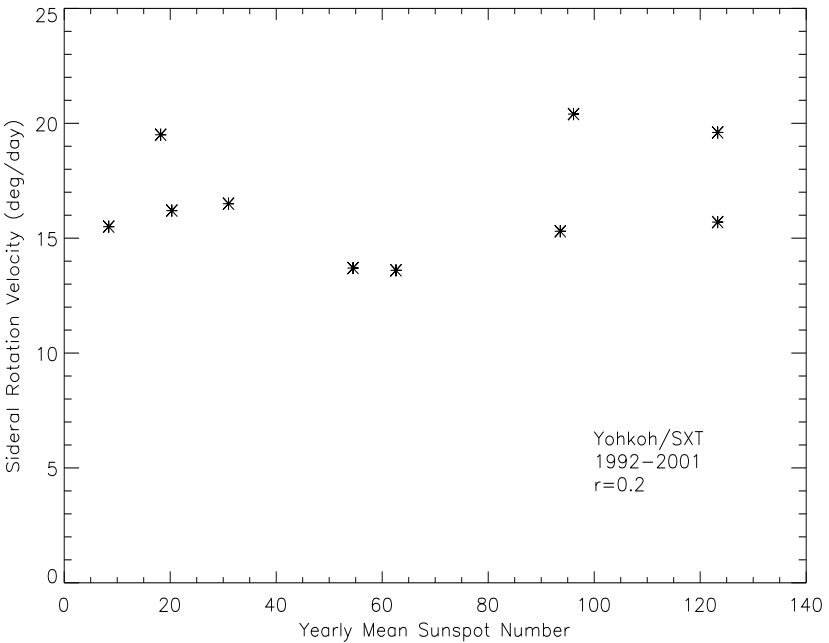

Fig. 4. Coronal sidereal angular rotation velocity (derived from XBPs of Yohkoh/SXT full-disc images) is compared with Yearly Mean Sunspot Number for the years from 1992 to 2001. The correlation coefficient $(r)=0.2$.

A part of the Yohkoh/SXT data analysis was done at NAOJ \& ISAS when the author held an NAOJ Visiting Professorship in 2002. The author would like to express his sincere thanks to Tetsuya Watanabe and Takashi Sakurai for the discussion, their kind hospitality, and support during his stay at NAOJ. Many thanks go to Katharine Reeves and Y. Katsukawa for their help in accessing the Hinode/XRT and Yohkoh/SXT images. The author is grateful to Ed DeLuca and L. Golub for their valuable suggestions and discussion of the XRT observations and results. Thanks go to B. A. Varghese for his help at many stages during the analysis of X-ray full-disc images and to A. Satyanarayanan for reading the manuscript and giving valuable suggestions. The author is thankful to the anonymous referee for many constructive comments and suggestions on the paper. 


\section{References}

Balthasar, H., Vázquez, M., \& Wöhl, H. 1986, A\&A, 155, 87

Brajša, R., Wöhl, H., Vršnak, B., et al. 2001a, A\&A, 374, 309

Brajša, R., Vršnak, B., Ruždjak, V., et al. 2001b, in Recent Insights into Physics of the Sun and Heliosphere: Highlights from SOHO and Other Space Missions, ed. P. Brekke, B. Fleck, \& J. B. Gurman, ASP Conf. Ser., 200, IAU Symp., 203, 377

Brajša, R., Wöhl, H., Vršnak, B., et al. 2002a, A\&A, 392, 329

Brajša, R., Wöhl, H., Vršnak, B., et al. 2002b, Sol. Phys., 206, 229

Brajša, R., Wöhl, H., Vršnak, B., et al. 2004, A\&A, 414, 707

Golub, L., \& Vaiana, G. S. 1978, ApJ, 219, L55

Golub, 1., \& Pasachoff, J. M. 1997, The Solar Corona (Cambridge, UK: Cambridge University Press)

Golub, L., Krieger, A. S., Silk, J. K., Timothy, A. F., \& Vaiana, G. S. 1974, ApJ, 189, L93

Golub, L., Krieger, A. S., Harvey, J. W., \& Vaiana, G. S. 1977, Sol. Phys., 53, 311
Hara, H., \& Nakakubo-Morimoto, K. 2003, ApJ, 589, 1062

Harvey, K. L. 1996, in Magnetic Reconnection in the Solar Atmosphere, ed. R. D. Bentley, \& J.T. Mariska, ASP Conf, Ser., 111, 9

Kambry, M., \& Nishikawa, J. 1990, Sol. Phys., 126, 89

Karachik, N., Pevtsov, A. A., \& Sattarov, I. 2006, ApJ, 642, 562

Kariyappa, R., \& Varghese, B. A. 2008, A\&A, 485, 289

Krieger, A. S., Vaiana, G. S., \& Van Speybroeck, L. P. 1971, in Solar Magnetic Fields, ed. R. Howard, IAU Symp., 43, 397

Longcope, D. W., Kankelberg, C. C., Nelson, J. L., \& Pevtsov, A. A. 2001, ApJ, 553,429

Mehta, M. 2005, Bull. Astr. Soc. India, 33, 323

Nakakubo, K., \& Hara, H. 1999, Adv. Space Res., 25, 1905

Sattarov, I., Pevtsov, A. A., Hojaev, A. S., \& Sherdonov, C. T. 2002, ApJ, 564, 1042

Vaiana, G. S., Krieger, A. S., Van Speybroeck, L. P., \& Zehnfennig, T. 1970, Bull. Am. Phys. Soc., 15, 611

Vats, H. O., Cecatto, J. R., Mehta, M., Sawant, H. S., \& Neri, J. A. C. F. 2001, ApJ, 548, L87

Zhang, J., Kundu, M., \& White, S. M. 2001, Sol. Phys., 88, 337 